\title{
The lateral neck radiograph for an impacted fish bone in the aero-digestive tract: Going back to basics
}

\author{
Dushyant Shetty, David AT Gay \\ Department of Clinical Radiology, Plymouth Hospitals NHS Trust, Plymouth, UK \\ Email: dushyant.shetty@nhs.net
}

Received 6 October 2012; revised 15 November 2012; accepted 22 November 2012

\begin{abstract}
Aim: To evaluate the radio-opacity of fish bones from a number of species using digital radiography in order to establish whether advances in acquisition and interpretative techniques have affected the radiologist's ability to detect impacted fish bones. Methods: The bones from six species of fish commonly consumed in the United Kingdom were radiographed using a soft tissue neck phantom by means of a digital radiographic X-ray tube. The images were looked at by 15 radiology consultants and registrars who determined whether the bones were visible or not using General Electric (GE) PACS workstations. Results: The radiographed bones from all six species of fish were visible by all $15(100 \%)$ radiology registrars and consultants. Conclusion: Digital radiogramphy and modern PACS workstations have meant that fish bones can be visualized irrespective of species. The lateral neck radiograph therefore may still have an important role in the investigation of impacted fish bones in the aerodigestive tract.
\end{abstract}

Keywords: Digital Radiography; Radio-Opacity; Fish Bones

\section{INTRODUCTION}

Ingestion and impaction of a foreign body (FB) is a common reason for an acute presentation to hospital [1]. Whilst children account for up to $80 \%$ of foreign body ingestion or impaction [2], the most commonly ingested foreign bodies in adults are fish bones [3]. Most impacted fish bones can be readily identified and retrieved under direct vision, however a number of patients require radiological investigation for localization. A lateral soft tissue neck radiograph remains the first line investigation to aid localization.

Literature from the early 1990s reported varying degrees of accuracy with which a lateral soft tissue neck radiograph could reliably be used to diagnose an impac- ted fish bone [1]. Sundgren et al. [4] suggested that $71 \%$ of bones were missed on X-ray, while Singh et al. [3] found $48 \%$ of fish bones to be radiolucent and therefore potentially non-diagnostic. Both these studies however, were performed using plain film radiography and not digital radiography, the latter of which is now universally used in medical imaging for healthcare today.

Fish bones most commonly lodge in the oropharynx, especially the tonsillar fossa and posterior third of the tongue [5]. Localization of bones in these areas is relatively straightforward using pharyngeal mirrors or flexible nasopharyngoscopes. It is when impaction occurs at less common sites, such as at the cricopharyngeus or cervical oesophagus that radiological investigation has a role to play. It is in this location that impaction most often leads to perforation [1]. Furthermore, in this location symptoms and signs are often unreliable and clinical examination can be challenging. It is here that a radiologically assisted diagnosis is required early in order to avoid potentially fatal complications such as oesophageal perforation, retropharyngeal abscess formation, peri-oesophagitis, mediastinitis or vascular fistula formation [1]. Evans et al. called for the complete abandonment of the lateral neck radiograph in assessing for the presence of impacted fish bones [6]. Using phantoms of approximately equivalent soft tissue densities to the cervical region and standard exposure factors for a lateral neck radiograph they found a large proportion of fish bones to be of insufficient radio-density to be easily visible [6]. It is worth reiterating that the above study was performed using plain film radiography, whose image quality and spatial resolution might have a bearing on interpretation, not to mention that interpretation was performed without the use of modern-day visualization techniques that allows the radiologist to window images.

The visibility of fish bones is dependant on its inherent radio-density. Ell et al. postulated that a fish bone's density can be inferred from its functional design and habitat [7]. For example deep sea fish have reduced ossification of the skeleton and tend to be smaller than surface dwelling fish; making them relatively radio-lucent. Sur- 
face dwelling fish such as cod, on the other hand, are active swimmers exhibiting very little bone reduction and are therefore relatively radio-opaque [7].

This study aims to address the issues related to the visualization of bones from differing species of fish using digital radiography.

\section{MATERIALS AND METHODS}

The bones from six popular fish consumed in the United Kingdom (namely Bream, Plaice, Mackerel, Pollock, Bass and Trout) were sourced locally. Pollock was chosen because it is closely related in structure and habitual behaviour to Cod [8]. The bones were removed from fillets of fish and cleaned in water. A selection of bones from each species of fish were cooked, in order to establish whether cooking the bones had any effect on their radioopacity. Cooking was performed by grilling them in a conventional oven at between 180 and 220 degrees Celsius.

Bones from each species were measured and placed within a standardised soft tissue neck phantom. Radio graphs were then taken using the General Electric (GE) Definium 8000 digital radiographic X-ray tube with an exposure of $4.0 \mathrm{mAs}$ at $\mathrm{KV} \mathrm{80,} \mathrm{which} \mathrm{are} \mathrm{the} \mathrm{standard}$ exposure factors used for lateral neck soft tissue radiography.

The images were reviewed by 15 radiology consultants (6) and registrars (9) from Plymouth Hospital's NHS Trust on a GE PACS workstation. The bones were either visible or not on the lateral neck radiograph and tabulated on a results sheet.

\section{RESULTS}

Twelve fish bones from six different species of fish were imaged to assess their radio-opacity (Figure 1). The bones were either cooked or uncooked. The average length of the bones was $24.8 \mathrm{~mm}$ with a range of $20 \mathrm{~mm}$ to $31 \mathrm{~mm}$. All twelve radiographs containing fish bones were visible and indicated as such by the 15 radiology registrars or consultants. Both cooked and uncooked bones were equally visible on the radiographs. There were 6 (40\%) radiology consultants and $9(60 \%)$ radiology registrars. There was one control film containing no bones and this was correctly identified by all 15 registrars and consultants.

\section{DISCUSSION}

There is ongoing debate as to the potential role of plain film radiography in the initial assessment of patients presenting with an impacted foreign body. With fish bones remaining a significant culprit [3], the importance of early detection in order to avoid the potential complications alluded to earlier is paramount. As stated by Palme

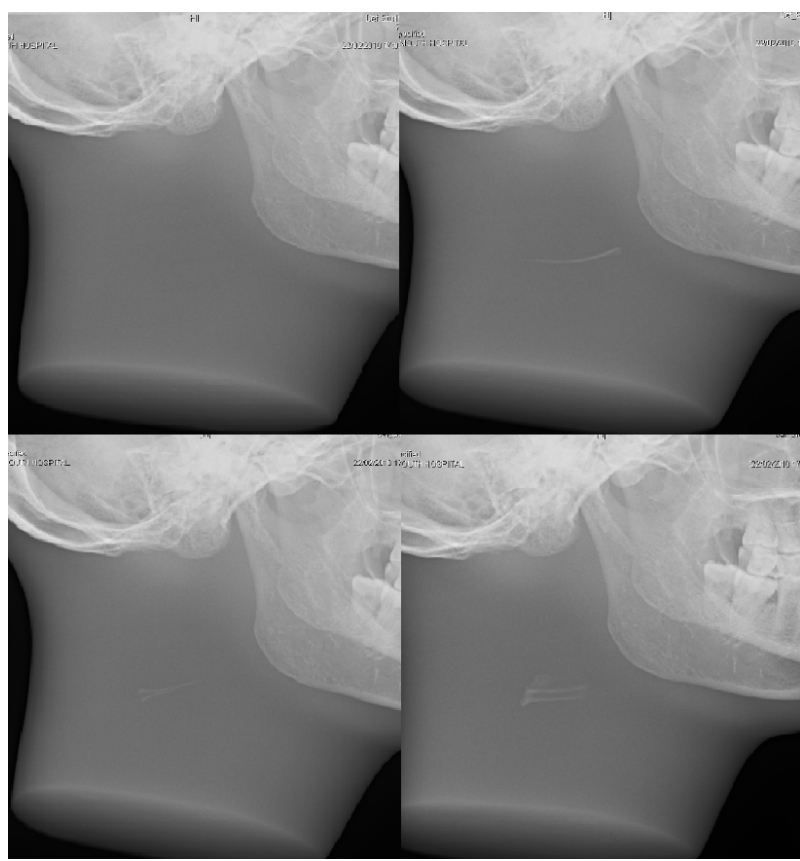

Figure 1. Digital radiography of fish bones in a standard soft tissue neck phantom. Clockwise from top left image; control image, Pollock, Bream and Bass.

et al. [1], the authors are in total agreement that computed tomography (CT) is far superior to plain film radiography in both the detection and accurate localization of a fish bone as well as the recognition of any complications. Given the evidence collected by this study however, the authors suggest that plain film radiography may still have a role as a first line investigation.

The differing radio-opacities of fish bones have been documented previously $[7,9]$. As outlined in the introduction, the radio-density of a fish bone is thought to be inferred from the intrinsic functionality of the fish in question. Ell and Sprigg [7] concluded that trout and mackerel were poorly visualized by soft tissue radiographic techniques and thus knowledge of both the fish ingested and the relative radio-opacities of various fish would be useful in film interpretation. The use of digital radiography and PACS software with windowing capabilities however, as demonstrated by this study, has revealed that all six fish species including those of trout and mackerel were visible. The authors are unfortunately unable show a direct visualization comparison of digital radiography and conventional plain film radiography, as used by Ell and Sprigg, because our institution like many in the United Kingdom stopped using traditional filmscreen radiography almost ten years ago. Furthermore both cooked and uncooked bones were visualized equally suggesting that there was no difference in the ability to interpret bones depending on whether they were cooked or not. Kirkham and English [10] suggested that cooking may alter the radio-opacity of fish bones; this does not 


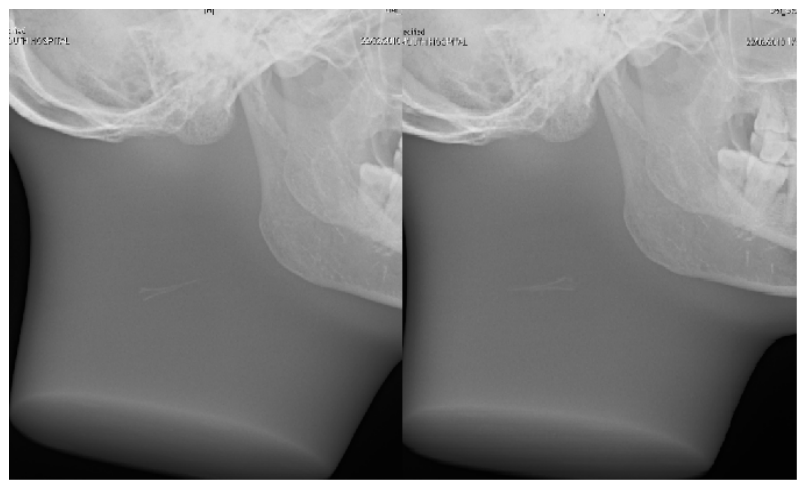

Figure 2. Digital radiography of cooked (left) and uncooked (right) Bass.

seem to be the case when using modern acquisition and interpretation techniques (Figure 2).

The diagnosis of foreign bodies within the neck, particularly bones can be potentially awkward due to the ossification of the cricoid, thyroid or hyoid cartilages [10-12]. The authors do not suggest that the findings of this study mean that the absence of a radio-opaque foreign body on a lateral soft tissue neck would completely allay the radiologists' fears of an impacted fish bone. This is because fish bones could still be obscured by the hyoid bone and laryngeal cartilages. This study has shown that fish bones, be they cooked or uncooked, fresh water or sea water fish can be identified with digital radiography. Further studies using $\mathrm{CT}$ as the potential gold standard and phantoms containing structures mimicking the hyoid bone and laryngeal cartilages need to be undertaken.

The authors believe that the lateral neck radiograph may still have a role to play in the investigation of an impacted fish bone in the aero-digestive tract. It is a relatively low dose examination that owing to the advances both in the quality of radiographic images produced and in the software used by radiologists for image interpretation has meant that fish bones regardless of species are of sufficient radio-opacity to be visible using digital radiography.

\section{ACKNOWLEDGEMENTS}

The authors thank Mr. Jason Hubbard for his assistance in taking the radiographs and Ticklemore Fish Shop, Totnes for supplying the fish bones.

\section{REFERENCES}

[1] Palme, C.E., Lowinger, D. and Peterson, A.J. (1999) Fish bones at the cricopharyngeus: A comparison of plain-film radiography and computed tomography. The Laryngoscope, 109, 1955-1958. doi:10.1097/00005537-199912000-00011

[2] Deeba, S., Purkayastha, S., Jeyarajah, S. and Darzi, A. (2009) Surgical removal of a tea spoon from the ascending colon, ten years after ingestion: A case report. Cases Journal, 2, 7532.doi:10.4076/1757-1626-2-7532

[3] Singh, B., Kanto, M., Har-El, G. and Lucente, F.E. (1997) Complications associated with 327 foreign bodies of the pharynx, larynx and oesophagus. Annals of Otology, Rhinology, and Laryngology, 106, 301-304.

[4] Sundgren, P.C., Burnett, A. and Maly, P.V. (1994) Value of radiography in the management of possible fish bone ingestion. Annals of Otology, Rhinology, and Laryngology, 103, 628-631.

[5] O’Flynn, P. and Simo, R. (1993) Fish bones and other foreign bodies. Clinical Otolaryngology, 18, 231-233. doi:10.1111/j.1365-2273.1993.tb00837.x

[6] Evans, R.M., Ahuja, A., Rhys, W.S. and Van Hasselt, C.A. (1992) The lateral neck radiograph in suspected impacted fish bones- does it have a role? Clinical Radiology, 46, 121-123. doi:10.1016/S0009-9260(05)80316-2

[7] Ell, S.R. and Sprigg, A. (1991) The radio-opacity of fish bones- species variation. Clinical Radiology, 44, 104-107. doi:10.1016/S0009-9260(05)80506-9

[8] Partridge, B.L. (1982) Structure and function of fish schools. Scientific American, 246, 114-123. doi:10.1038/scientificamerican0682-114

[9] Ell, S.R. (1989) Radio-opacity of fishbones. Journal of Laryngology and Otology, 103, 1224-1226. doi:10.1017/S0022215100111417

[10] Kirkham, N. and English, R. (1984) I have a bone stuck in my throat. British Journal of Medicine, 289, 423-424.

[11] Richardson, G.S. (1955) Unusual calcification of cricoid cartilage masquerading as a foreign body in the oesophagus. Archives of Otolaryngology, 62, 316-318. doi:10.1001/archotol.1955.03830030082015

[12] Zoller, H. and Bowie, E.R. (1957) Foreign body in the air and food passages versus calcifications of laryngeal cartilages. Archives of Otolaryngology, 65, 474-478. doi:10.1001/archotol.1957.03830230050010 